\title{
FORENS IC TEXTILE FIBRE MATCHING BY MULTIRESOLUTION PLANNING AND COLOUR SPACE PATTERN RECOGNITION
}

\author{
G. J.McEwen and A.J.Wi $1 \mathrm{k}$ inson \\ Electrical and Electronic Engineering \\ The Queen's University of Belfast
}

Colour is a key element in the discrimination and matching of textile fibres for forensic purposes. Two aspects of research aimed at developing an autamated forensic textile fibre matching system by colour computer vision are outlined. The segmentation procedure is described. It is based on a multiresolution image format and incorporates progressive plan refinement. Filters for building the multiresolution image are compared and recommendations made as to when they might be useful. The use of colour, as it relates to this application, is formulated in terms of classical pattern recognition. The colour space clusters associated with textile fibre classes are described and a suitable clustering scheme is presented.

\section{INTRODUCT ION}

Colour has been incorporated in computer vision systems with a number of different reasons in mind. Usually the meaningful partitioning of colour imagery is the sole objective, being performed by combining achromatic and chromatic information ${ }^{12}$. Others extend this aim and use colour as an auxilliary aid to object recognition and label1ing so that it becomes a component of the scene description as an additional adjective ${ }^{3}$. Closer to the purpose of the research described in this paper is the important place thà multispectral information takes for region classification in remote sensing (with spatial context, temporal and external auxilliary information only adopting a secondary role) ${ }^{4}$. The goal has shifted to the recognition of specific objects or regions where colour is the primary discriminatory feature, the objects or regions being otherwise similar. The colour of an object may signal its state ${ }^{5}$, code its property ${ }^{6}$, or as in this research, identify its class membership. Colour is a key element in textile fibre matching for forensic purposes.

Textile fibres are amongst the number of trace materials which may remain as evidences after the conmission of a crime, being transferred from the criminal's clothing to the victim or the surroundings, or vice versa. Whilst combing the scene of the crime the forensic scientist will recover as many of these fibre artefacts as possible, perhaps also removing at the same time a large number of superfluous fibres. Furthermore, a small sample of fibres, denoted the control fibres, are isolated as being representative of a suspect's garments. The forensic scientist seeks to establish whether the suspect was at the scene of the crime by searching for fibre matches whilst comparing the recovered fibres with the control fibres thoroughly. The fibres are initially compared under a microscope on the basis of their colour. This is the most significant visual discriminatory feature at the low magnifications used during the preliminary sift. Putative matches are isolated and forwarded for more detailed confirmatory tests.

Research is being carried out with the aim of developing a colour vision system which will replace the preliminary manual screening procedure, locate and facilitate the relocation and removal of matches from amongst recovered fibres. This application shares functional elements common in many other machine vision tasks. These include (colour) image preprocessing and conditioning, image segmentation, object discrimination and identification and scene description. In this paper two elements will be emphasized. First, the segmentation procedure. Although tailored to the imagery (fibre microscopy), the details are pertinent to those applications, (or general vision systems), where objects of interest form a small part of the image, but are seperable from the 'background, on the bas is of a few image properties. Second, the object discrimination and matching process, which is based on object colour, will be formulated in terms of classical pattern recognition. The account is relevant to those applications where colour has a primary role in object indentification, or to generel vision systems where colour has a subordinate role but is useful for certain tasks.

\section{SEGMENTATION BY MULT IRESOLUTION PLANNING}

In common with the imagery associated with many tasks involving bright field microscopy which may be tackled by computer vision, the imagery associated with fibre analysis usually consists of small regions of focal interest, strands in this instance, separated to a greater or lesser degree by irrelevant 'background'. Such imagery is not confined to microscopy and is

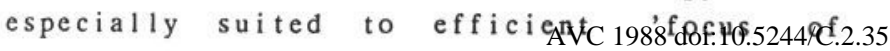


attention, processing using a multiresolution image representation? Global decisions regarding the partitioning of the high resolution image may be made by examining condensed versions of it. Given the foregoing, a segmentation procedure based on multiresolution techniques has been adopted in this research.

Briefly, in multiresolution image processing an image is represented at several degrees of spatial detail simultaneously. This structure will be referred to as a pyramid. The prototype image, denoted level 0 , having $2^{n} \times 2^{n}$ cells, is recursively decomposed into $2^{n-1} \times 2^{n-1}, \ldots, 2 \times 2,1 \times 1$ versions, labelled leve 1 to level $n$ [fig.1.]. Generally, level k $(0 \leqslant k \leqslant n)$ has $2^{n-k} \times 2^{n-k}$ cells. In this research, the cell at location $(i, j)$ on level k holds the result of a linear or non-linear filter operation defined on a window of side $s$, ( odd or even), at level k-1, having origin

$(2 \mathrm{i}-\operatorname{ceil}[\mathrm{s} / 2]+1,2 \mathrm{j}-\operatorname{ceil}[\mathrm{s} / 2]+1)$

(ceil[x] rounds $x$ up to the nearest integer number). This determines the correspondences between the cell lattices at adjacent pyramid levels for odd and even filter windows as shown in fig.2. In general, a cell at level k represents $\left((s-1) 2^{\mathrm{k}-m}-(\mathrm{s}-2)\right)^{2}$ cells at level $m(m<k)$. Each level may consist of several planes, each containing a different point property. In colour imagery, three planes might hold the red, green and blue (RGB) colour component images. An individual point attribute is thus uniquely addressed by its plane, level and spatial coordinate.

Various segementation schemes utilising this multiresolution format have been suggested in the literature. This research has used a
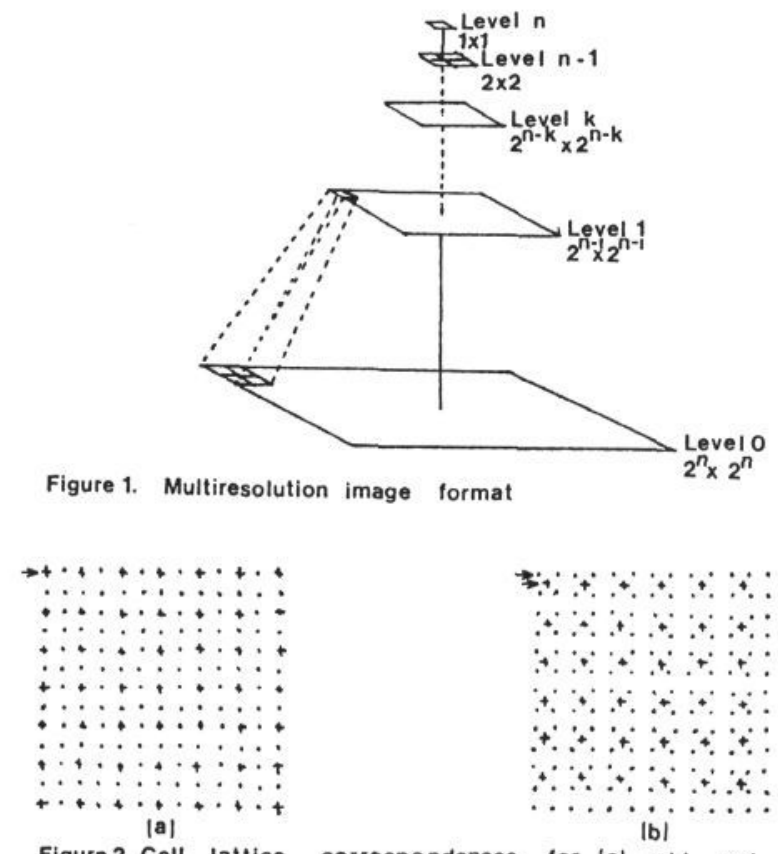

Figure 2. Cell lattice correspondences for $|a|$ odd and (b) even filter windows between adjacent pyramid levels. Crosses refer to level $k$ and dots to level $k-1$. Origins indicated by arrows. strategy incorporating 'planning' similar to that proposed by Kelly ${ }^{8}$. Rather than recursively descending the pyramid to acheive segmentation ${ }^{2}$, or iteratively building a pyramid in which cells from adjacent levels are linked in a way that embodies the segmentation ${ }^{10}$, the segmentation is successively planned in greater detail whilst descending the pyramid level by level. The plan at a particular level is stored in a special data structure which can grow or shrink as the plan is extended or disolved. It is used to direct the formation of a more spatially refined plan at the next lower level so that at level 0 the plan embodies the segmentation, delineating those parts of the image of particular interest. This staightforward scheme is suited to a serial implementation and avoids the excessive (and unnecessary) cost of recursive computation (by trading memory and elegance for speed) and incorporates the idea of 'focus of attention, in a more relevant way than iterative pyramid linking (by avoiding the cost of 1 inking those parts of the image of little or no interest).

\section{Filters for Pyramid Building}

The inherent flexibility afforded by using software to build the pyramid has been exploited by investigating the suitablity of a selection of linear and non-linear filters for pyramid building. This is not a complete study and is essentially empirical but it does serve to illustrate the importance of considering the nature of a pyramid in terms of how appropriate it is for the particular task. In building a pyramid the spatial resolution is reduced whilst those image details and properties of special utility are preserved. Thin strands, characteristic of fibre microscopy, are important to segmentation and must be propagated throughout the pyramid. The size and type of filter used for pyramid building will influence the nature of the pyramid, so as to determine the degree to which important details are retained at higher levels; details which, in this case, affect the performance of the segmentation process.

As a preliminary it is necessary to isolate those aspects of the task which might be influenced by the character of a pyramid. In our research the segmentation of the fibre imagery is based on a simple thresholding of the point intensity at successive pyramid levels. This procedure is based on the assumption that peaks in the grey level histogram correspond, in the main, to coherent regions in the image. This assumption may be valid when dealing with a restricted image domain (where we make implicit use of our knowledge of its properties) and particularly in a controlled environment (in which we can 
arrange lighting and sensor geometry, for example). When adopting this approach and when using multiresolution techniques it is important that the integrity of the spatial and dynamic distributions of grey levels be preserved at successively higher pyramid levels, since this is the key to segmentation. An ineffective summary of the prototype image at higher levels, manifesting itself by disruption to their grey level histograms, will degrade the performance of the segmentation.

\section{Grey Level Pdf Preservation:} An Indicator of Filter Suitablity

Given the foregoing we have employed the grey level histogram (or more accurately, the grey level probability distribution function ( $p d f$ )) as the basis on which to compare filters for pyramid building. The pdf, by its nature, is a normalised global characteristic of an image, although widely different images may be described by the same pdf (which implies that a comparison of pdfs is not a faithful, or in any way a complete, distance metric for images). The filter judged 'best' is that which consistently builds pyramids in which the pfds of the images at level $k,(0<k \leqslant n)$, are most similar to that of the level 0 image. This approach has been adopted because the pdf is of fundumental importance to the segmentation scheme. We assume that those changes to an image as it is consolidated (eg blurring), which are in some sense relevent to the quality of the segmentation, are echoed by changes to the pdf.

\section{A Measure of Distance Between Pdfs}

Since we have selected the pdf as the most pertinent aspect of a pyramid (for our task) a suitable measure of the disparity between the pdf at level $k$ and, that at level 0 is needed. One possibility is the vector distance between their cumulative distribution functions ${ }^{11}$ (cdfs).

Let $X$ be a discrete random varible which can take values $x_{i},(0 \leqslant i<g)$, where $x_{i}$ are the grey level values, and $g$ is the total number of grey level values. Let $N_{k}$ denote the number of cells at level $k$ and $n_{k}(i)$, the number of cells at level $k$ having grey level $x_{i}$, so that

$$
N_{k}=\sum_{i=0}^{g-1} n_{k}(i)
$$

Define the probability of grey level $x_{i}$ for a particular image as

$$
P\left\{X=X_{i}\right\}_{k}=n_{k}(i) / N_{k}
$$

and denote it $p_{k}(i)$. The values $p_{k}(i)$, $(0 \leqslant i<g)$, constitute the pdf for that image. Obviously

$$
\sum_{i=0}^{g-1} p_{k}(i)=1
$$

The value of the cdf at level k, denoted $c_{k}(i)$, being the probability of grey level less than or equal to $x_{i}$, is given by

$$
P\left\{X \leqslant x_{i}\right\}_{k}=\sum_{j=0}^{i} p_{k}(j)
$$

The vector distance $d_{k}$ between the cdf at level $k$, and that at level 0 is then

$$
d_{k}=\sum_{i=0}^{g-1}\left|c_{k}(i)-c_{o}(i)\right|
$$

Werman et al ${ }^{11}$ illustrate in the introduction to their paper how this measure is a better distance metric for one dimensional histograms than the straightforward vector distance between pdfs, (computed by substituting $p_{k}$ (i) and $p_{0}(i)$ for $c_{k}(i)$ and $c_{o}(i)$ in (5)), which fails to take into account the similarity between those feature descriptors $x_{i}$ which are near each other.

\section{The Candidate Filters}

We limited our comparison, on the basis of the foregoing criteria, to the following linear and non-linear, odd and even sided, filters.

$2 \times 2$ unweighted average and $4 \times 4$ unweighted average : The $2 \times 2$ average filter is popular, being especially suited to the pyramidal format (since the image is consolidated by powers of 2). The $4 \times 4$ unweighted average has also been used before, finding application as a first iteration in the linked pyramid building procedure.

$4 \times 4$ weighted average: The weighted average filter after Burt ${ }^{12}$ (see also Meer et a ${ }^{13}$ ), is a generic type of notable flexability. The form of the filter kernel, defined by its weights, is determined by the size of the filter and a single parameter, a (adopting Burt's notation). We included two filters, having $a=0.37$ and $a=0.58$. The former approximates convolution by a Gausian when applied repeatedly, characteristically reducing high frequency noise (but at the cost of blurring). The latter, in contrast, is not unimodal. It is characterised by inferior high frequency noise at tenuation.

$2 \times 2$ average-median: In an at tempt to preserve some of the useful chacteristics of the median filter (which operates on a square window with odd side) the $2 \times 2$ average-median averages those cell values ranked second and third subsequent to ranking all four cells in the window according to grey level magnitude.

$3 \times 3$ median: The median filter is based on the fact that when the values of the cells comprising the window are ranked in order of magnitude, irrespective of their position within the window, any deviant values are located at the extremities and that value which best represents the window occupies the median position. 
$5 \times 5$ FIR-median hybrid $(\mathrm{fmh})^{14}$ : This adaptable operator intrinsically retains high frequency details, such as lines, whose orientations are determined by FIR-subfilter masks which constitute the filter. The $5 \times 5$ fmh selected for this experiment is referred to as 2LH in Nieminen's notation and has eight bidirectional FIR-subfilters of the averaging t ype.

\section{The Images}

Our account here concerns three images [fig.3.a,b, c]. Each has $256 \times 256$ pixels at level 0 .

Image [a] : A computer generated image comprising of 5 concentric semi-circles of widths $1,2,3,4$ and 5 pixels with grey level 64, against a uniform background with grey level 128. This image was used to evaluate, for each of the filters, the extent to which edges are disrupted and details are erased, and is especially useful since the form of its

\section{Figure 3.}
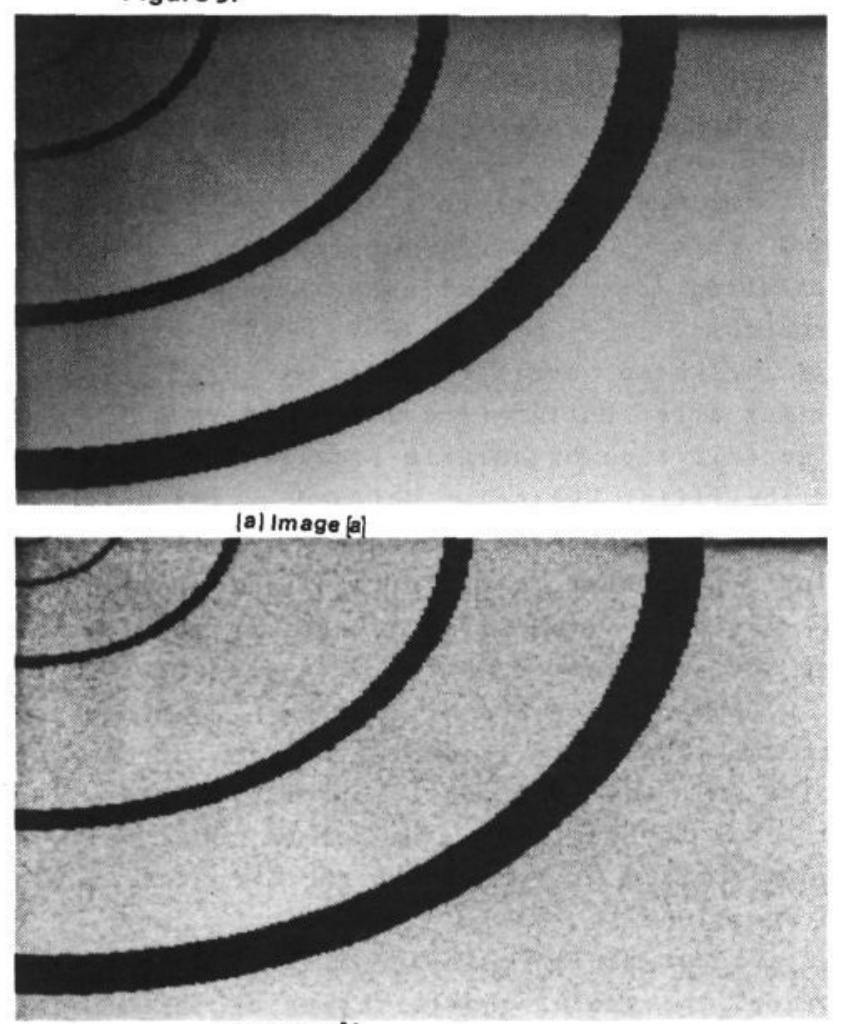

(b) Image $|b|$

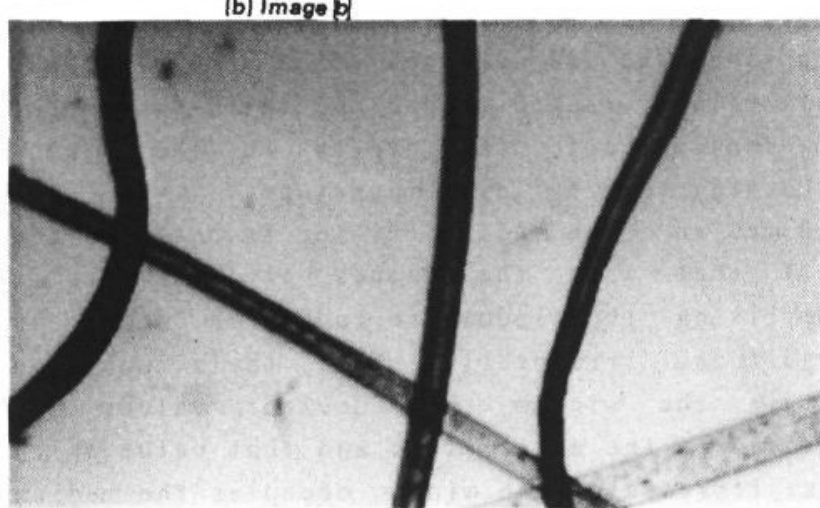

$[c]$ Image $[c]$ content resembles that of textile fibre imagery.

Image [b] : A similar image derived by disrupting the former with additive Gausian noise with variance $=9.051$. Noting that our metric $d_{k}$ responds similarly to degradation due to edge effects, and enhancement due to noise reduction (since both change the pdf), this image was used to evaluate the relative importance at tached to each of these effects.

Image [c] A microscope image of red, green and blue textile fibres viewed through a $x 4$ objective lens and a red optical filter (Schott SFK15).

For images [a] and [c] the metric $d_{k}$ was calculated from the pdfs at levels 1 to 4 inclusive, relative to the pdf of their level 0 counterparts. The metric $d_{k}$ was computed at levels 1 to 4 of image [b] relative to level 0 of images [a]. Fig.4.a,b,c is a graphical summary of the variation of $d_{k}$ for increasing $k$, for the images included here.

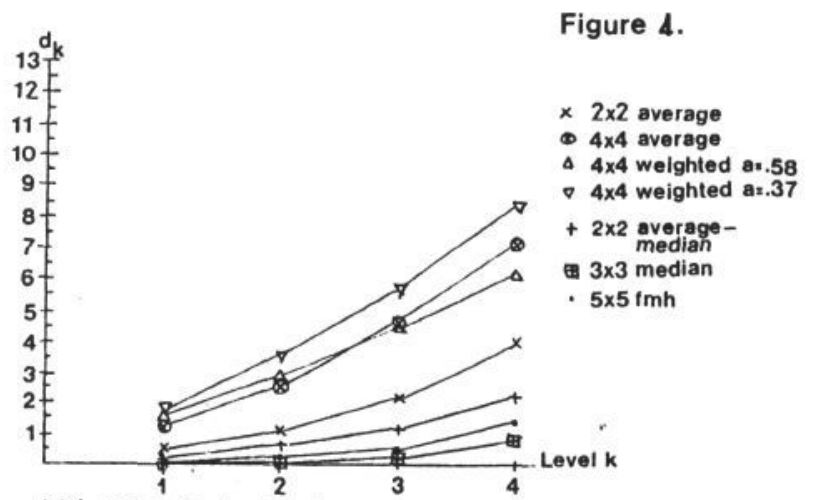

(a) $d_{k}$ at levels $k=1$ to 4 , image la

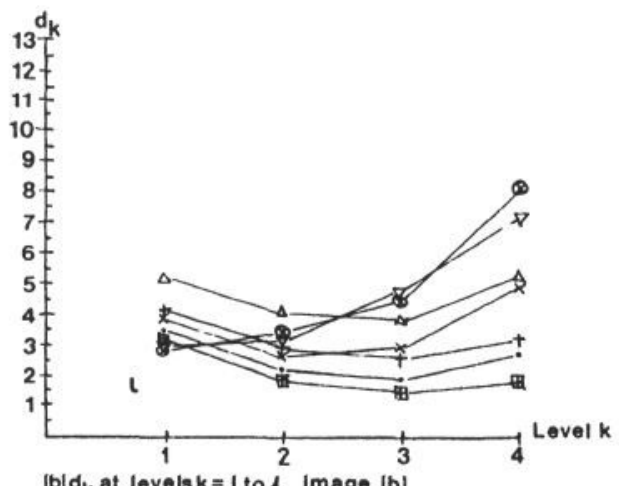

$|b| d_{k}$ at leveis $k=\mid$ to 4 , Image $|b|$

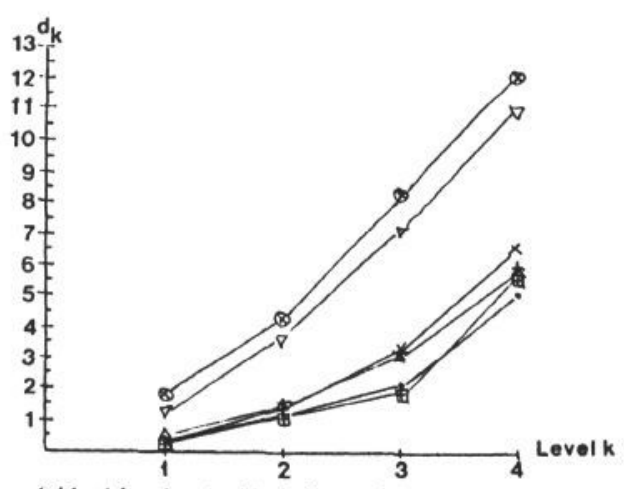

$|c| d_{k}$ at levels $k=\mid$ to 4 , image $|c|$ 


\section{Observat i ons}

For image [a] it was observed that the $4 \times 4$ unweighted average and the $4 \times 4$ weighted average with $a=0.37$ blurred edges extensively (as was expected). This explains consistently high values of $d_{k}$ for thesefilters, so that they are ranked 'worst' [fig.4.a]. Most significantly, from our point of view, the two most narrow rings were smeared so as to be indistinguishable from each other at level 3 for both filters. Fig4.a also shows that the $4 \times 4$ unweighted average with $a=0.58$ performs badly. This can be attributed, not to blurring, but to an oscillating effect apparent at edges. This artefact remains because the weights of the filter kernel do not decrease monotonically from the centre towards the edge. Blurring, although present, was less evident for the $2 \times 2$ unweighted average, and 1 ess so for the $2 \times 2$ average-median; an observation also reflected in fig.4.a. The $2 \times 2$ average-median did 'break' rings however, so that the most narrow was no longer evident at level 4. This behaviour, characteristic of the median operation, was most clearly exhibited by the $3 \times 3$ median filter. This filter, followed by the $5 \times 5 \mathrm{fmh}$, performed consistently best according to the criteria. Significantly, however, the $3 \times 3$ median operation removed the most narrow ring so that it was no longer present at level 1. Moreover, the trend continued, so that the most narrow ring evident at level k was no longer present at level $\mathrm{k}+1$. The $5 \times 5$ fmh preserved horizontal, vertical and diagonal lines better than the $3 \times 3$ median (consistent with its expected behaviour), but nevertheless 'broke' and eventually removed rings. To a large extent these observations were also true of image [c] [fig.3.c and fig. $4 . c]$.

Concerning image [b], it was visually apparent that the $4 \times 4$ unweighted average and the $4 \times 4$ weighted average with $a=0.37$, reduced noise best, but as has been noted, they blurred edges most severely. From their ranked position in fig.4.b it is obvious that our measure $d_{k}$ penalises blur to such an extent that restorative 'gains' due to noise reduction are swamped by the detrimental 'losses' due to edge and detail disruption. Noting that $d_{k}$ does not fall monotonically for any of the filters, it may be deduced that the point at which $d_{k}$ starts to rise again is that at which edge and detail disruption become significant, in the sense that they outweigh noise reduction.

\section{Dis cus s i on}

Spatial details are necessarily erased at higher levels of a pyramid. The linear filters examined here blur details, and the non-linear median-type filters 'ignore' details. Consider a compact object, small relative to the filter window. If the object is to be detected and recorded at the next level it must fall, at least partly, inside a window. The number of windows which overlap an object is dependent on the window size and the object's location. Horizontally and vertically adjacent windows overlap each other so that they share $s^{2}-2 s$ cells, and diagonally adjacent windows share $\mathrm{s}^{2}-4 \mathrm{~s}+4$ cells. Whether the presence of the object is recorded at the next level is determined by the degree to which each of these windows overlaps the object, and the type of operator. The amount of overlap is a function of object location. Consider for example a $2 \times 2$ object having its origin at an odd coordinate position. If the windows are also $2 \times 2$ it will be partly overlapped by four windows. A $2 \times 2$ unweighted average operator will respond equally (weakly) to the presence of the object at all four locations. In contrast, a $2 \times 2$ average-median operator will not record the object at any of the four locations. This is sometimes called 'falling through the cracks'. If the $2 \times 2$ object has its origin at an even coordinate position both $2 \times 2$ operators will respond at only one location; that which completely covers the object. Similar arguments hold for 1 inear and non-linear filters with odd windows. The level at which useful processing can commence is directly influenced by the size and type of filter used for pyramid building.

Given the limited nature of this invesigation we can make the following observations, valid for tasks where grey level integrity is important. For imagery where details are of little consequence, the $3 \times 3$ median or the $5 \times 5$ FIR-median hybrid are recommended. The nature of fibre microscopy, characterised as it is by thin lines, dictates that either the $2 \times 2$ average or $2 \times 2$ average-median be used, these being less severe on details than the former pair. The same conclusion extends to imagery where a focal region is small relative to the size of the image. Given the foregoing, the $2 \times 2$ average-median filter has been employed in this research for building multiresolution structures.

\section{FI BRE DISCRIMINATION BY COLOUR SPACE PATTERN RECOGNITION}

The literature reports how colour space clusters corresponding to spectrally homogeneous regions in a scene $c$ an be used as the basis of segmentation ${ }^{25,26}$. Alternatively, chromatic information supplements or replaces achromatic and is used as a measure of the similarity beween spatially proximate points, determining whether they are co-members of a corporate region. 
Using colour solely for segmentation, however, belittles its value. The objective in this research is to use colour to discriminate between, identify and match textile fibres; objects which are otherwise similar. The previous section reported that achromatic information is sufficient to segment the imagery, given its domain, but full chromatic information is required to achieve the further objective. The problem can be formulated in terms of pattern recognition.

Traditionally, unsupervised pattern recognition is conveniently subdivided into two tasks, training and classifying. Given a set of measurements taken from class exemplars, the former is concerned with the partitioning of that heterogeneous data set into mutually exclusive and collectively exhaustive subsets, or the partitioning of the measurement space into mutually exclusive regions, in a way which reflects the division of objects into disjointed classes: The latter denotes the operation of assigning objects of unknown origin to classes on the basis of their measurements and the results of the training phase. In this context, textile fibres correspond to the objects. The underlying premise is that fibre classes are spectrally dissimilar to an extent that they have associated with them separable colour space clusters. Fibres of unknown origin should be classified as belonging to one of the control fibre classes, or indeed, none of the control fibre classes, given a set of colour measurements and a previously partitioned colour space. The point RGB colour components

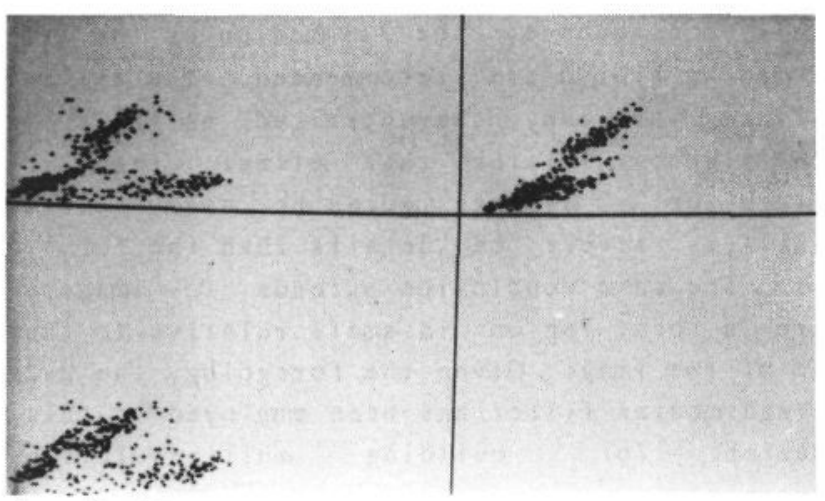

Figure 5. RGB space clusters for red, green and blue fibres

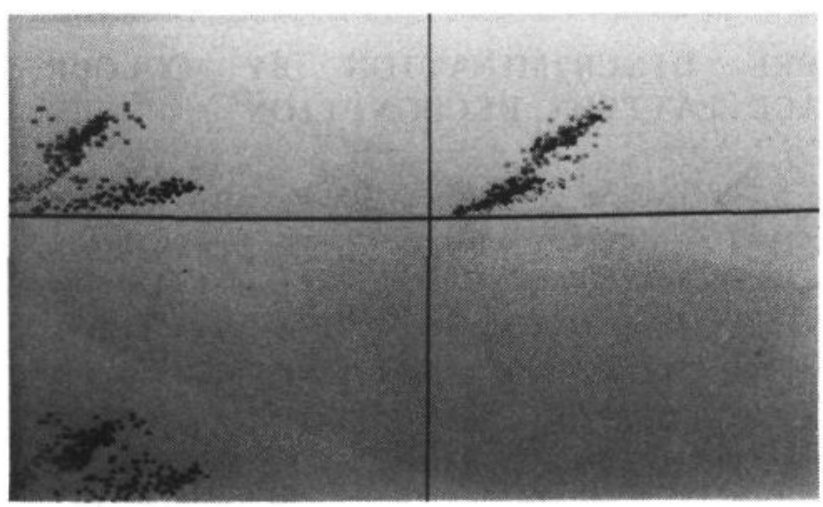

Figure 6. Clusters partitioned by Koontz clustering algorithm (or some transformation thereof ${ }^{1}$ ) constitute the colour measurements, and map directly on to a 3-dimensional colour space.

The heterogeneous training set is formed by sampling points randomly from the fibre regions of segmented images of mixed control fibre exemplars. The clusters illustrated in fig.5. are typical of those derived from fibre imagery. The high degree of correlation apparent between the colour components is exhibited by clusters which resemble elongated ellipsoids aligned towards the colour space origin. The clusters are not 'we1l behaved'. Multivariate analysis has shown that they are non-normal, being particularly affected by out liers. Colour component variance tends to be large, so that clusters are smeared. These reflect the nature of the fibres which are chacteristically not uniformly coloured, of ten having darker edges. They may also be artefacts of the segmentation procedure and the quality of the imagery. This, of course, militates against clustering and classifying based on colour information alone. Nevertheless evidence suggests that the clusters are separable for different fibre classes and procedures devoloped thus far have been successul in so $f$ ar as heterogeneous training sets can be partitioned sat isfactorily.

Automatic partitioning of the training set is acheived by employing a graph-theoretic clustering algorithm after Koontz et a1 ${ }^{17}$, supplemented by two of the subcluster agglomeration criteria of Mizoguchi et a $1^{18}$. This scheme is non-iterative and non-parametric. It is based on a non-parametric estimate of colour space point density, partitioning the training set by linking colour space points to one of their nearest neighbours along the 1 ine of 1 argest positive density gradient. It is capable of detecting clusters of arbitrary shape and size, its usefulness having been verified by its successful application to multispectral remote sensing dataio. Fig.6. illustrates the partitioning of the previous data set based on a k nearest neighbour density estimate with 12 nearest neighbours.

Samples derived from images of fibres of unknown origin are presented for classification based on the training set partition. Samples largely falling within one of the control fibre clusters signal that the fibre from which they are derived is a member of the corresponding control fibre class. The implementation of a technique for acheiving this is part of ongoing research. 


\section{CONCLUS ION}

In the foregoing, two aspects of research have been detailed concerning the development of a colour vision system capable of sifting a large sample of textile fibres removed from the scene of a crime, searching for, locating, and facilitating the relocation of those fibres which match exemplar fibres removed from a suspect's garments, on the basis of their colour.

The sparse nature of the imagery associated with fibre microscopy suggested that a multiresolution image format might be advantageous, allowing the development of efficient 'focus of attention' segmentation techniques. A scheme based on progressive plan refinement has been adopted, segmenting the imagery by thresholding point intensity. A selection of multiresolution pyramid building filters were compared quantitatively and qualitatively to determine their relative suitabilty for application to fibre imagery. Given the limited extent of the investigation, a $2 \times 2$ average-median filter was chosen. This filter exhibits good detail and edge retention. This was reflected by the degree to which the dynamic and spatial distributons of grey levels were maintained at successive pyramid levels. Two non-linear filters were of note. The $3 \times 3$ median filter and the $5 \times 5$ FIR-median hybrid filter performed well but tended to obliterate details.

Colour is a key element in textile fibre matching, and its value was noted in the wider field of computer vision. If it is to become an integral part of computer vision it must be incorporated not only for the purpose of segmentation or of discrimination (as is the case in this research), but in ways similar to its uses in the human visual system which can detect colour edges, perceive colour regions, use colour as a qualifying adjective, search and identify using colour cues, and perceive form and motion taking colour as an auxilliary indicator ${ }^{20}$. In the context of this research, the discriminatory problem was formulated in terms of pattern recognition. The colour space clusters associated with fibre classes, although illustrated as being 'badly behaved', are separated to a sufficient degree to permit fibre matching to be carried out by colour space pattern recognition. A mode-seeking clustering algorithm was presented as being suitable to partition a heterogeneous training set made up of samples from mixed fibre exemplars. This paves the way for a classification scheme to identify fibres of unknown origin, recording their locations in a composite of concise scene descriptions which will facilitate the relocation of matching fibres. Further research will concentrate on the coupling of the segmentation and classification phases, with a goal to acheive fibre discrimination in multiple resolutions.

\section{REFERENCES}

1. Ohta, Y-I. ; Kanade, T. ; Sakai, T. 'Color Information for Region Segmention' Comput . Graphics and Image Process 13, (1980) $222-241$

2. Chang, B-H. ; Kim, S-D. ; Kim, J-K. 'Threshold Selection Algorithm for Extracting a Uniform Colour Region in an Image' Electron. Let t. Vol 3, (1987), 25, $1362-1363$

3. Ohta, Y-I. 'Knowledge Based Interpretation of Outdoor Scenes' Marshfie1d MA ; Pitman Publishing Inc. (1985)

4. Tailor, A. ; Cross, A ; Hogg, D.C. ; Mason, D.C. 'Knowledge-based interpretation of remotely sensed images, Image and Vision Comput. Vol 14, 2, 67-83 (1986)

5. Keller, J.M. ; Covavisaruch, N. ; Unklesbay, K. ; Unklesbay, N. 'Color Image Analys is of Food' Proc. IEEE Conf. on Computer Vision and Pattern Recognition $619.621,(1986)$

6. Kelly, R.B. ; Faedo, W. 'A First Look into Color Vision' Proc. SPIE. Int. Soc. Opt . Eng. (USA) 579, 96-103 (1985)

7. Li, Z-N. ; Uhr, L. 'A Pyramidal Approach for the Recognition of Neurons Using Key Features, Pattern Recognition Vol 19, $(1987), 1,55.62$

8. Kelly, M.D.' 'Edge Detection in Pictures by Computer Using Planning, Machine Intelligence Vol 6, (1971), 379.409

9. Shapiro, L.G. 'Data Structures for Picture Processing : A Survey' Comput. Graphics and Image Process. 11, (1979), 162-184

10. Antonisse, H.J. 'Image Segmentation in Pyramids' Comput. Graphics and Image Process. 19, (19े82), 367-383

11. Werman, M. ; Peleg, S. ; Rosenfeld, A. 'A Distance Metric for Multidimensional Histograms' Comput. Vision, Graphics and Image Process. Vol 32, 3, (1985), 328-336

12. Burt, P.J. 'Fast Filter Transforms for Image Processing, Comput. Graphics and Image Process. 16, (1981), 20-51 
13. Meer, P. ; Baugher, E.S. ; Rosenfeld, A. 'Frequency Domain Analys is and Synthes is of Image Pyramid Generating Kernels, IEEE Trans. on Pattern Analysis and Machine Intelligence Vol PAMI-9, (1987), 4, 512-522

14. Nieminen, A. ; Heinonen, P. ; Neuvo, Y. 'A New Class of Detail-Preserving Filters for Image Processing, IEEE Trans. on Pattern Analysis and Machine Intelligence Vol PAMI-9, (1987), 1, 74-90

15. Schacter, B.J. ; Davis, L.S. ; Rosenfeld, A. 'Scene Segmentation by Cluster Detection in Color Spaces' SIGART Newsletter 58, (1976), 16-17 (1976)

16. Coleman, G.B. ; Andrews, H.C. 'Image Segmentation by Clustering' Proc. IEEE 67, $(1979), 5,773-785$

17. Koontz, W.L.G. ; Narendra, P.M. ; Fukunaga, K. 'A Graph-Theoretic Approach to Nonparametric Cluster Analys is, IEEE Trans. on Computers Vol C-25, 9, (1976), 936-944

18. Mizoguchi, R. ; Shimura, M. 'Nonparametric Algorithm for Detecting Clusters Using Hierarchical Structure IEEE Trans. on Pattern Analysis and Machine Intelligence Vol PAMI -2, (1980), 4, 292-300

19. Narendra, P.M. ; Goldberg, M. 'A Non-Parametric Clustering Scheme for LANDSAT' Pattern Recognition Vol 9, (1977), 207-215

20. Gershon, R. 'Aspects of Perception and Computation in Color Vision' Comput. Vision Graphics and Image Process. Vo1 32, 3, (1985), 244-277 